\section{Detection of liver metastases in cancer patients with geographic fatty infiltration of the liver: the added value of contrast-enhanced sonography}

\author{
Tommaso Vincenzo Bartolotta, Adele Taibbi, Dario Picone, Andrea Anastasi, \\ Massimo Midiri, Roberto Lagalla \\ Department of Radiology-Di.Bi.Med., University of Palermo, Palermo, Italy
}

Purpose: The aim of this study is to assess the role of contrast-enhanced ultrasonography (CEUS) in the detection of liver metastases in cancer patients with geographic liver fatty deposition on greyscale ultrasonography (US).

Methods: Thirty-seven consecutive cancer patients ( 24 women and 13 men; age, 33 to 80 years; mean, 58.1 years) with geographic liver fatty deposition, but without any detectable focal liver lesion on greyscale US, underwent sulphur hexafluoride-enhanced US. Two readers reported by consensus the presence, size, and location of any detected lesion. All patients underwent magnetic resonance imaging (MRI) as a confirmatory study. Sensitivity, specificity, positive and negative predictive values (PPV and NPV), and accuracy were calculated.

Results: Seven focal liver lesions (size, 4 to $10 \mathrm{~mm}$; mean, $6.1 \mathrm{~mm}$ ) were detected in 4/37 patients (10.8\%): four metastases (size, 5 to $10 \mathrm{~mm}$; mean, $6.7 \mathrm{~mm}$ ) were detected both by CEUS and MRI, with one hemangioma and two cysts (size range, 4 to $6 \mathrm{~mm}$; mean, $5.3 \mathrm{~mm}$ ) detected by MRI only. In 1/37 patients (2.7\%), CEUS misdiagnosed geographic fatty change as three metastases. In 32/37 patients (86.5\%), no lesions were detected. Sensitivity, specificity, PPV, NPV, and accuracy of CEUS were 100\% (95\% confidence Interval $[\mathrm{Cl}], 1.000$ to 1.000), $97.1 \%(95 \% \mathrm{Cl}, 0.914$ to 1.027$), 75 \%, 100 \%$, and $97.3 \%$, respectively. No statistically significant differences were found between CEUS and MRI in the detection of focal liver lesions $(P=0.480)$, whereas both of them performed better than baseline US $(P<0.001)$.

Conclusion: CEUS improves the detection of liver metastases in cancer patients with geographic liver fatty deposition on greyscale US.

Keywords: Fatty liver; Liver diseases; Ultrasonography; Contrast media; Liver neoplasms; Neoplasm metastasis

\section{Introduction}

The liver is a very common site for the spread of malignancy, and follow-up programs for patients with cancer, including liver imaging performed by means of transabdominal ultrasonography (US), are

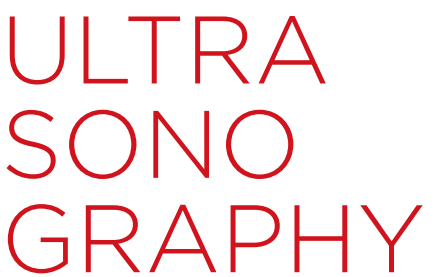

ORIGINAL ARTICLE

https://doi.org/10.14366/usg.16041 pISSN: 2288-5919 e elSSN: 2288-5943 Ultrasonography 2017;36:160-169

Received: October 6, 2016

Revised: October 29, 2016

Accepted: November 16, 2016

Correspondence to:

Tommaso Vincenzo Bartolotta, MD, PhD, Department of Radiology-Di. Bi.Med., University of Palermo, Via del Vespro, 129, 90127, Palermo, Italy

Tel. $+39-338-5941227$

Fax. +39-091-6552325

E-mail: tv_bartolotta@yahoo.com

This is an Open Access article distributed under the terms of the Creative Commons Attribution NonCommercial License (http://creativecommons.org/ licenses/by-nc/3.0/) which permits unrestricted noncommercial use, distribution, and reproduction in any medium, provided the original work is properly cited.

Copyright (C 2017 Korean Society of Ultrasound in Medicine (KSUM)

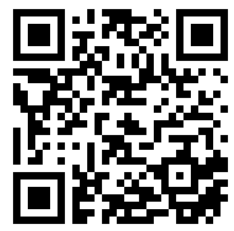

How to cite this article:

Bartolotta TV, Taibbi A, Picone D, Anastasi A, Midiri M, Lagalla R. Detection of liver metastases in cancer patients with geographic fatty infiltration of the liver: the added value of contrast-enhanced sonography. Ultrasonography. 2017 Apr;36(2):160-169. 
reported to improve survival [1].

Nevertheless, in clinical practice US may show equivocal results, with a sensitivity ranging from $40 \%$ to $80 \%$ according to the diameter of lesions and the experience of the sonologist [2,3]. Fatty infiltration of the liver, often induced by chemotherapy, may further worsen US performance in this crucial task [4]. In particular, unusual and heterogeneous forms of fatty deposition, such as geographic liver fatty deposition, may prevent greyscale US from reliably ruling out the presence of liver metastases in cancer patients, even in the absence of any overt and/or suspect focal liver lesion [5]. More recently, the introduction of contrast-enhanced ultrasonography (CEUS) has led to a dramatic improvement in diagnostic accuracy of US in the detection of liver metastases, when compared to conventional greyscale US $[2,6]$. CEUS has also been reported to improve the characterization of focal hepatic lesions in patients with fatty liver and in diagnosing both focal fatty infiltration and sparing [7-9]. Hence, we hypothesized that CEUS might be an effective tool in the detection of liver metastases during follow-up of cancer patients with geographic liver fatty deposition, but without any evidence of metastatic disease of the liver on conventional greyscale US.

\section{Materials and Methods}

\section{Patient Population}

This prospective study was approved by the Ethics Review Board of our institution. All patients gave their full informed consent before the CEUS examination. The implemented procedure was in accordance with the Declaration of Helsinki [10].

Between June 2013 and December 2013, 37 consecutive patients (24 women and 13 men; age range, 33 to 80 years; mean, 58.1 years) were enrolled in this study. Enrolled patients were referred to our institution for an abdominal US for detection of liver metastases during postsurgical follow-up (interval range, 8 to 222 months; mean, 56.2 months) of pathologically proven primary malignancies. Liver CEUS examinations were performed immediately following the standard US examination that diagnosed the geographic fatty infiltration, on the basis of the following inclusion criteria: (1) the absence of any overt and/or suspect focal liver lesion on preoperative US, including lesions showing US features of simple cyst, typical hemangioma and/or typical focal fatty sparing; (2) the presence of geographic liver fatty deposition on greyscale US, defined as patchy areas of increased liver reflectivity without a mass effect and/or vessel displacement [5]. The following exclusion criteria were also considered: (1) incomplete visibility of the liver parenchyma on preoperative US due to intervening bowel gas or patient body habitus; (2) clinical history of previously detected liver metastases with US, computed tomography (CT), and/or magnetic resonance imaging (MRI); (3) clinical history of liver metastases previously treated by radiofrequency or surgical procedures; (4) presence of local or distant nodal metastatic spread; (5) positive tumoral serum markers; (6) presence of critical illness and/or severe heart disease; (7) presence of allergy to sulphur hexafluoride (SonoVue); and (8) lack of final diagnosis proved by means of an adequate standard of reference (SOR). No patients were excluded due to severe steatosis.

All studied patients had previously received chemotherapy or chemo-radiotherapy for primary cancer located outside the liver: breast ductal or lobular carcinoma $(n=18)$, colorectal adenocarcinoma $(n=14)$, gastrointestinal stromal tumour $(n=1)$, intestinal neuroendocrine tumour $(n=1)$, pleural mesothelioma $(n=1)$, endometrial adenocarcinoma $(n=1)$, and ovarian adenocarcinoma $(n=1)$. Also, all patients had undergone a previous imaging follow-up at our institution, which confirmed the absence of liver metastases and geographic liver fatty deposition.

\section{US Technique}

Two experienced radiologists (more than 15 years experience in CEUS of the liver), who were aware of the patients' clinical histories, performed US scanning by means of either a MyLab Twice (Esaote Biomedica, Genoa, Italy) or an iU22 unit (Philips Ultrasound, Bothell, WA, USA), both provided with a multifrequency convex array probe (CA541 1-8 MHz and C 5-2 MHz) and contrast-specific imaging software (Contrast-Tuned and Pulse Inversion, respectively). A baseline survey examination, including a colour/power and pulsed Doppler analysis, was performed. Once set, the US parameters, such as focal zone and time gain compensation, were not changed during the course of the study. The US contrast agent used in the present study was SonoVue (Bracco, Milan, Italy), which was injected intravenously as a $2.4 \mathrm{~mL}$ bolus (equivalent to a $0.003 \mathrm{~mL} / \mathrm{kg}$ for $70 \mathrm{~kg}$ body weight) followed by $10 \mathrm{~mL}$ of normal sterile saline flush by using a 20- or 22-gauge peripheral intravenous cannula. A low frame-rate $(5 \mathrm{~Hz})$ and a very low mechanical index, ranging from 0.05 to 0.08 , were used for real-time imaging. One focus was positioned below the level of the lesion. Two consecutive examinations in the same session were performed with an interval time of at least 15 minutes to allow for contrast clearance of the previous contrast medium injection. The first examination, encompassing the right lobe of the liver, was performed with a subcostal approach and the patient lying on the left flank, whereas the second examination, encompassing the left lobe, was performed with an anterior abdominal approach and the patient lying supine. Each examination lasted about 5 minutes after bolus injection. Digital cineloops were registered both at baseline and during the whole post-contrast US 
scanning up to 5 minutes after the beginning of contrast agent bolus injection. All cineloops were digitally stored as raw-data on a PC-based workstation connected to the US units via a standard Ethernet link.

\section{On-Site Image Analysis}

Immediately after the completion of each CEUS procedure, the two examiners subjectively reviewed all cineloops offline but on screen, and evaluated by consensus the dynamic enhancement pattern of liver parenchyma and reported the presence, size, and location (according to the Couinaud classification system) of any detected lesion. For the purposes of this study, which is focused on cancer patients, any hypoechoic solid lesion (darker than the surrounding liver) in the late phase was considered malignant, since all metastases show this feature at CEUS, without any reported exception [11].

\section{Off-Site Image Analysis}

Two independent and experienced radiologists (with more than 10 years experience in the use and interpretation of (EUS) randomly reviewed all cineloops offline but on screen. Neither reader was involved in the scanning, and they were blinded to the final diagnosis, as well as to the identification, clinical histories, and other imaging findings of the patients.

The examinations were randomly and independently assessed in separate sessions. The readers were asked to evaluate the US in the same fashion as previously described for on-site image analysis.

\section{Standards of Reference}

All patients underwent MRI performed with a 1.5 T MR unit (Signa Excite, General Electric, Healthcare, Milwaukee, WI, USA) using a phased-array multicoil as a receiver coil. The MRI protocol included axial pre-contrast images acquired with T2-weighted fast-spin echo sequence (TR/TE, 4,000/76 msec; section thickness, 5-6 $\mathrm{mm}$ ) and T1-weighted axial in-phase and out-of-phase gradientrecalled-echo (GRE) sequence (TR/TE, 140/2.2-4.4 msec; section thickness, 5-6 mm). Dynamic studies were performed with threedimensional, fat-suppressed, T1-weighted GRE sequence (LAVA-TR/ TE, 3.8/1.2 msec; FA 12; slice thickness, 4.4 mm; intersection gap, 2 mm; FOV, 44 cm; matrix, 256×256) using a bolus-tracking system. Images were acquired on the axial plane immediately before and after intravenous injection of either $0.1 \mathrm{mmol} / \mathrm{kg}$ body weight of gadobenate dimeglumine (MultiHance, Bracco, Italy) at $2 \mathrm{~mL} / \mathrm{sec}$ or $0.025 \mathrm{mmol} / \mathrm{kg}$ body weight of gadoxetic acid at $1 \mathrm{~mL} / \mathrm{sec}$ through a 20-gage intravenous catheter by means of a power injector (Medrad Spectris Solaris EP MR Injection System, Bayer HealthCare, Wuppertal, Germany), followed by a 20-mL saline flush at the same injection rate. Scanning delays after automatic detection of contrast bolus were 18,60,180, and 300 seconds, respectively, for the acquisition of the arterial, portal-venous, 3-minute, and 5-minute phase. Hepatobiliary phases were obtained 120 minutes after the injection of contrast material, with the same scanning parameters. The interval between the MRI and the CEUS was 1-10 days (mean interval, 7 days).

Two experienced radiologists (with more than 15 years experience in MRI of the liver), not involved in the US scanning and not aware of clinical history, evaluated magnetic resonance (MR) examinations by consensus. Diagnostic criteria for liver metastases and fatty change of the liver at CEUS and MRI with hepatocellular specific contrast agent are reported in Tables 1 and 2, respectively [8,12-14].

\section{Statistical Analysis}

First, detection of liver metastases was analysed with patientby-patient analysis. The Wilcoxon signed rank test was employed to compare paired data from baseline US, CEUS, and contrastenhanced MR.

Second, detailed lesion-by-lesion analysis was performed and sensitivity, specificity (diagnostic performance) for baseline US, CEUS, and contrast-enhanced CT were compared by the two-tailed McNemar test with a continuity correction. A P-value $<0.05$ was

\section{Table 1. Diagnostic criteria for liver metastases}

Contrast-enhanced ultrasonography
- Hypovascular appearance in the extended portal-venous phase
regardless of contrast-enhancement behaviour in the arterial phase
Gd-BOPTA-enhanced magnetic resonance imaging
- Intermediately intense signal on T2-weighted images
- Hypointense signal on T1-weighted images
- Hypervascular appearance with diffuse enhancement or hypovascular
appearance peripheral rim-like enhancement at arterial-dominant
phase
- Hypovascular appearance at portal-venous and $2 \mathrm{hr}$ after Gd-BOPTA
injection

Gd-BOPTA, gadobenate dimeglumine.

\section{Table 2. Diagnostic criteria for fatty change of the liver}

\footnotetext{
Contrast-enhanced ultrasonography

- Area homogeneously iso-enhancing to liver parenchyma in all vascular phases

Gd-BOPTA-enhanced magnetic resonance imaging

- Area of increased signal on T1-weighted sequences; in-phase/out-ofphase: signal drop-out in out-of-phase imaging
}

Gd-BOPTA, gadobenate dimeglumine. 
considered to indicate a statistically significant difference in all tests.

Cohen's kappa was calculated to assess inter-operator agreement. Agreement was graded as poor $(\leq 0.20)$, moderate $(0.20$ to 0.40$)$, fair ( 0.40 to 0.60$)$, good $(0.60$ to 0.80$)$, or very good ( 0.80 to 1.00). Statistical analyses were performed using STATA/SE ver. 14 (StatCorp., College Station, TX, USA) and MedCalc (https://www. medcalc.org/calc/diagnostic_test.php).

\section{Results}

\section{Patient-by-Patient Analysis}

MRI confirmed the presence of fatty diffuse liver infiltration in all patients. Overall, according to our SOR (MRI), seven focal liver lesions (size range, 4 to $10 \mathrm{~mm}$; mean, $6.1 \mathrm{~mm}$ ) were detected in $4 / 37$ patients (10.8\%; age range, 43 to 70 years; mean, 53.3 years) (Table 3). Four metastases (size range, 5 to $10 \mathrm{~mm}$; mean, $6.7 \mathrm{~mm}$ ) were found in $3 / 37$ patients ( $8.1 \%$; age range, 43 to 70 years; mean, 55.4 years), one patient with breast carcinoma and two patients with colorectal adenocarcinoma; whereas three benign lesions - one hemangioma and two cysts (size range, 4 to $6 \mathrm{~mm}$; mean, $5.3 \mathrm{~mm}$ )-were detected in one of the already mentioned patients and in a fourth patient (Table 3). These four patients were followed up by means of US (interval range, 8 to 151 months; mean, 62 months), without any statistically significant difference from the

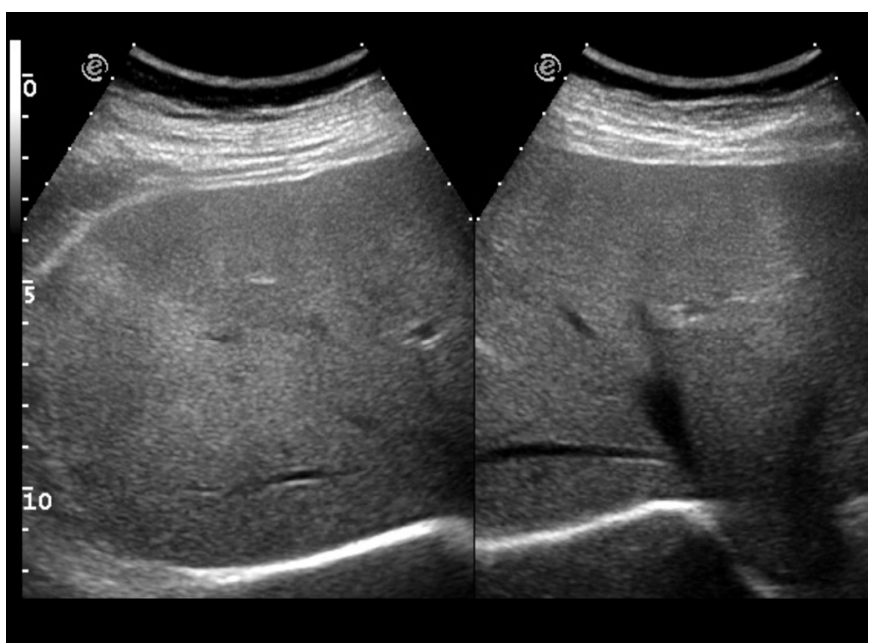

A

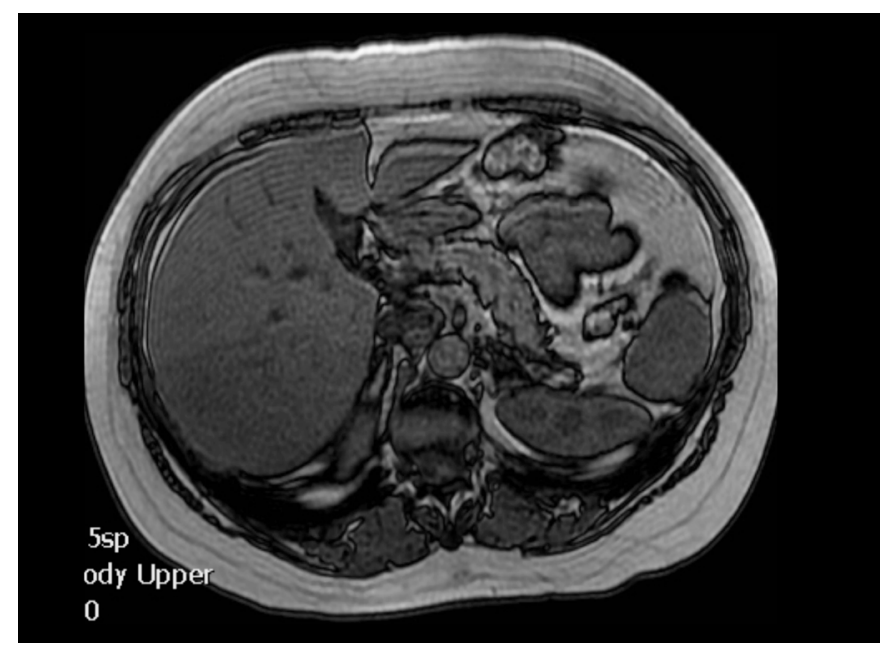

C

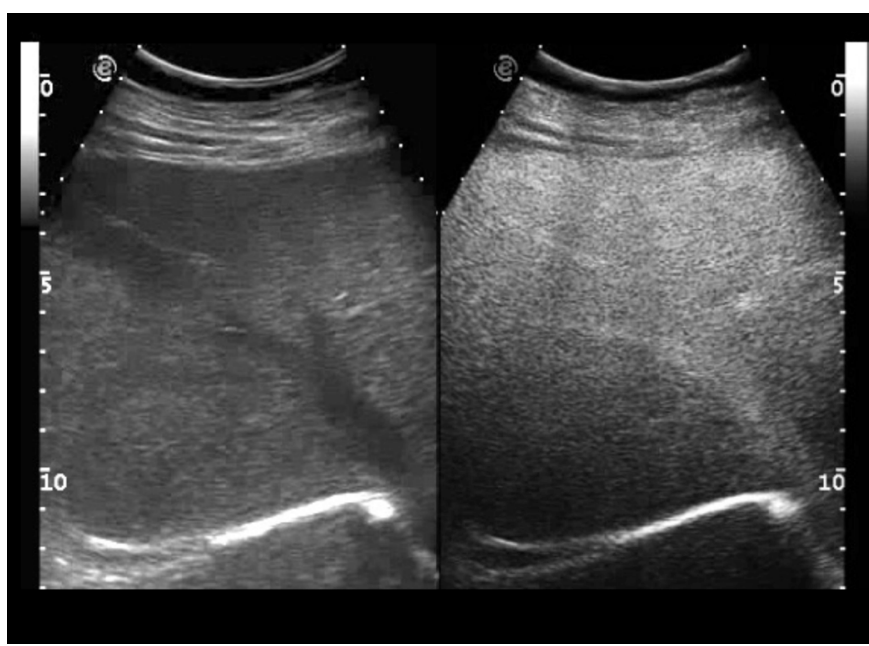

B

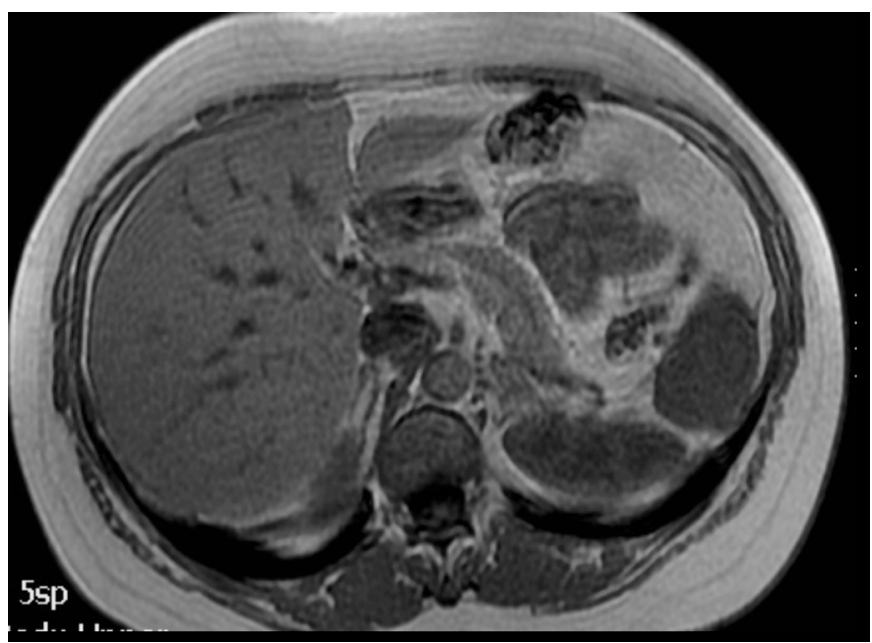

D

Fig. 1. A 53-year-old woman affected by breast cancer.

A, B. Oblique ultrasonography of the right hepatic lobe shows geographic fatty infiltration of the liver, but no evidence of any focal liver lesion either before (A) or after contrast medium injection (B) (right side). C, D. An unenhanced T1-weighted "out of phase" magnetic resonance imaging image confirms geographic fatty change of the liver, but absence of lesions by showing a clear-cut inhomogeneous signal drop-out of hepatic parenchyma, in comparison with the "in phase" T1-weighted image (D). 
Table 3. Liver lesions as detected by standard of reference

\begin{tabular}{ccccccl}
\hline Patient/sex/age (yr) & Cancer type & CEUS & MRI & Lesion size $(\mathrm{mm})$ & Liver segment & Lesion type \\
\hline 1/F/52.8 & CRC & + & + & 7 & IVa & Metastasis \\
1/F/52.8 & CRC & - & + & 4 & IVb & Simple cyst \\
2/M/70.3 & CRC & + & + & 10 & VII & Metastasis \\
2/M/70.3 & CRC & + & + & 5 & $\|$ & Metastasis \\
$3 / F / 43.2$ & Breast & + & + & 5 & IV & Metastasis \\
4/F/46.8 & Ovarian & - & + & 6 & $\|$ & Hemangioma \\
4/F/46.8 & Ovarian & - & + & 6 & III & Simple cyst \\
\hline
\end{tabular}

CEUS, contrast-enhanced ultrasonography; MRI, magnetic resonance imaging; F, female; M, male; CRC, colorectal cancer; +, detected; -, undetected.

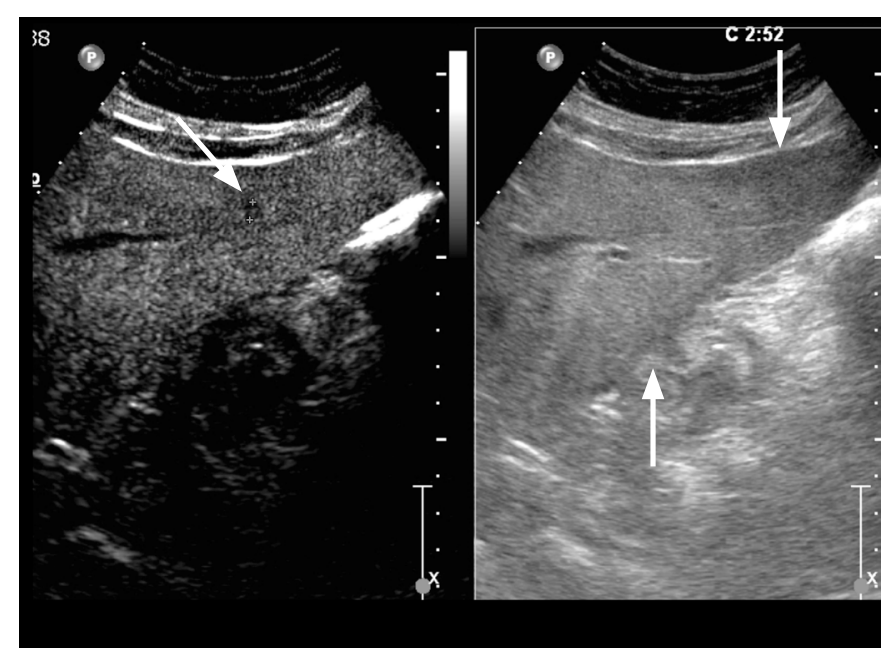

A

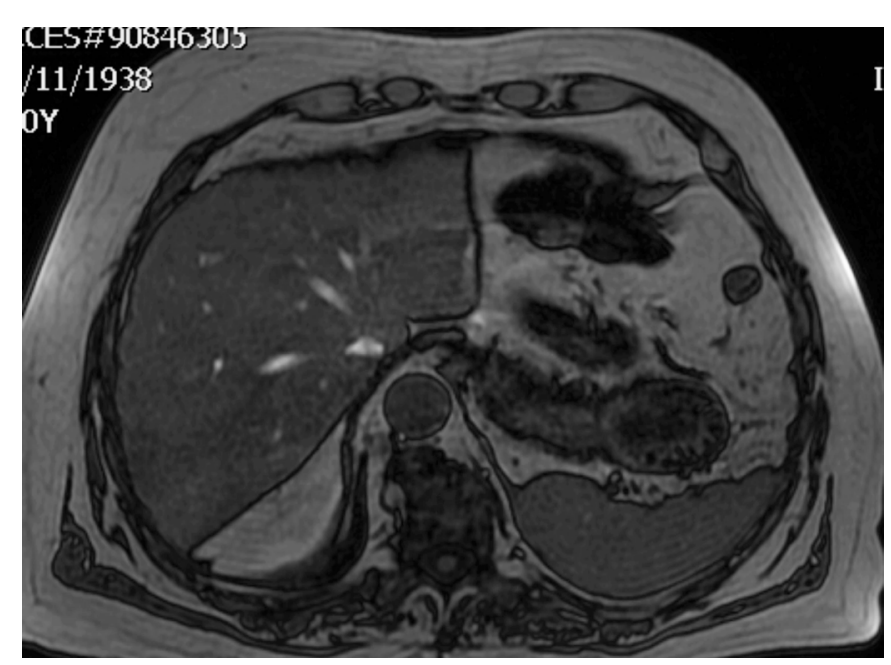

C

Fig. 2. Liver metastasis in a 70-year-old man (Table 3, patient 2).

A. Parasagittal B-mode ultrasonography of the left lobe (right side, arrows) shows geographic fatty change of the liver but no evidence of any focal liver lesion whereas contrast-enhanced ultrasonography (left side) depicts a $0.5-\mathrm{cm}$-sized hypoechoic focal liver lesion in segment II during extended portal-venous phase (calipers). B-D. "In phase" (B) and "out of phase" (C) unenhanced magnetic resonance (MR) T1weighted images confirm geographic fatty infiltration of the liver and contrast-enhanced MR T1-weighted image in the portal-venous phase reveals the hypovascular metastasis (D) (arrow).

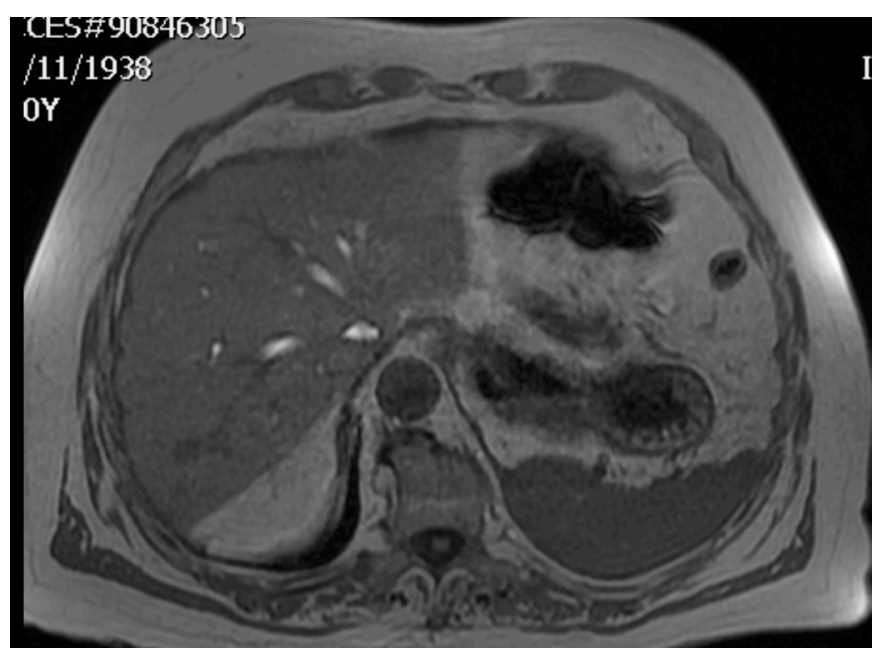

B

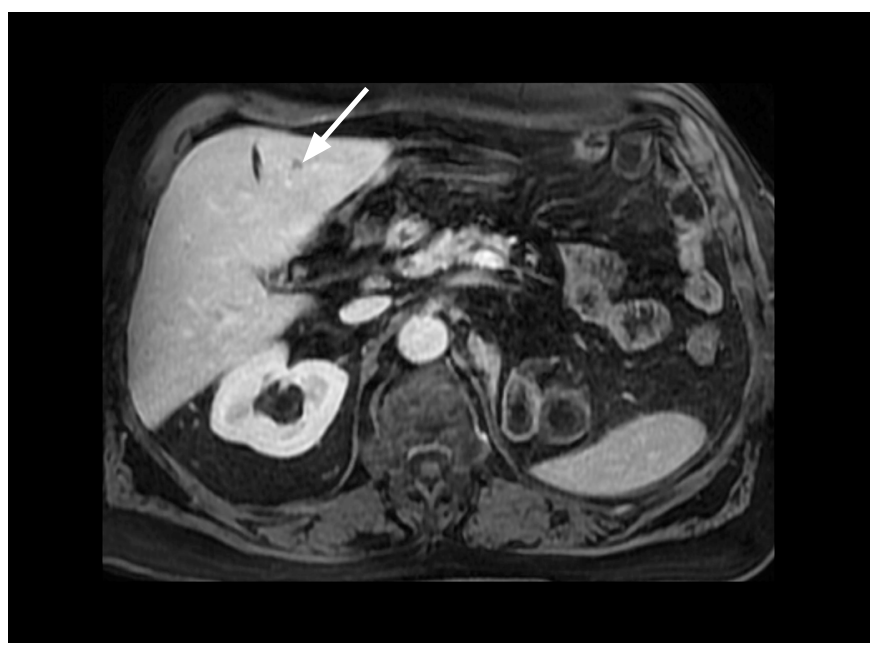

D 


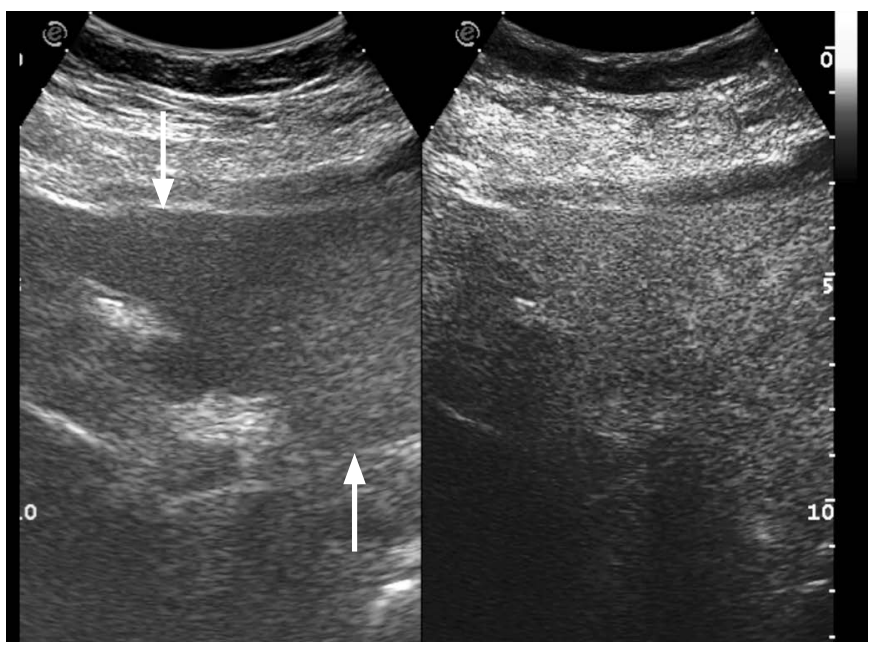

A

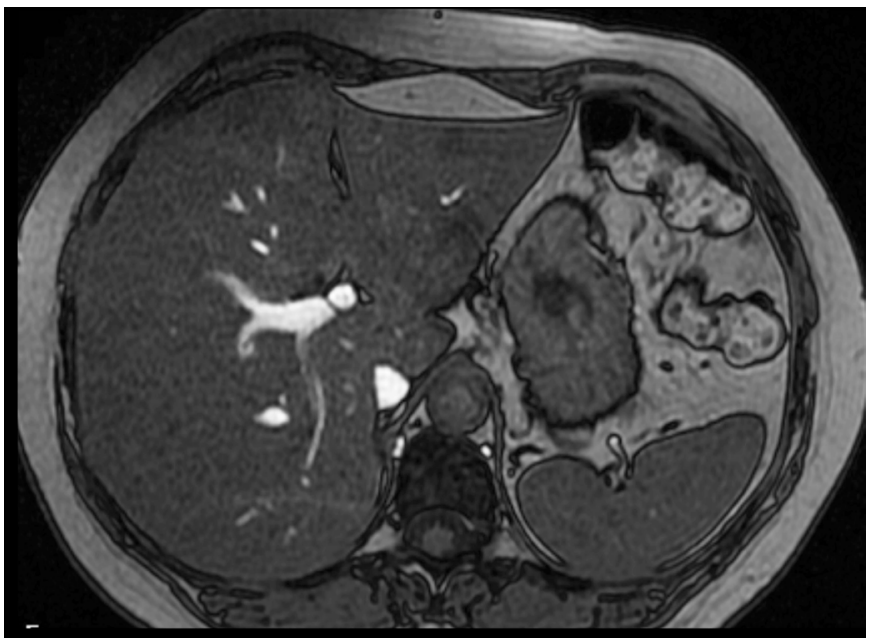

C

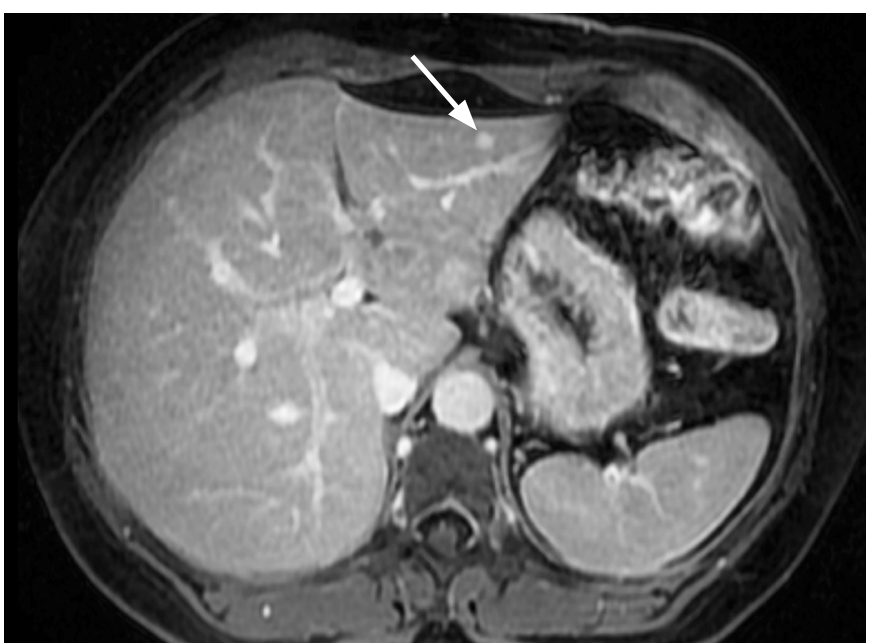

E

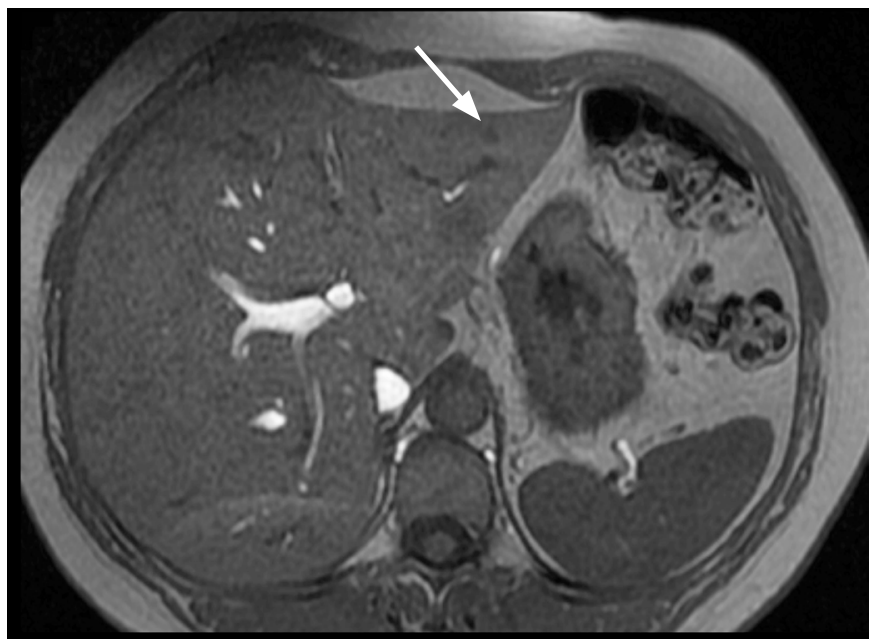

B

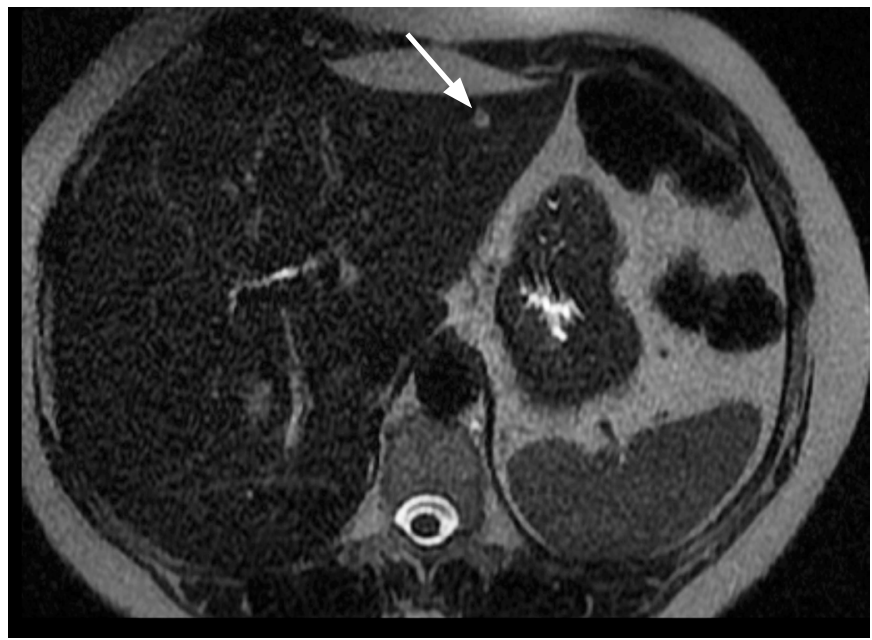

D

Fig. 3. Hemangioma in a 47-year-old woman (Table 3, patient 4). A. Transverse ultrasonography of the left liver lobe shows geographic fatty change without any detectable focal liver lesion either at $B$-mode ultrasonography (left side, arrows) or contrast-enhanced ultrasonography (right side). B, C. "In phase" (B) and "out of phase" (C) unenhanced magnetic resonance (MR) T1-weighted images confirm geographic fatty infiltration of the liver and reveal a hypointense 6-mm-sized lesion in segment II (arrow). D, E. The lesion is hyperintense on heavily T2-weighted MR image (D) and shows homogeneous contrast-enhancement on T1-weighted MR image in the late phase (E) (arrows). 


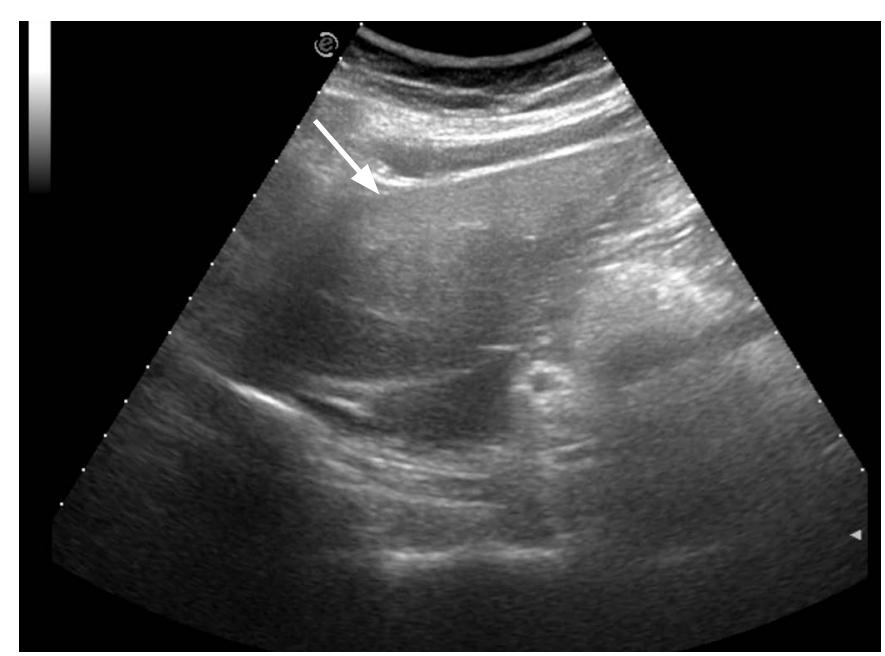

A

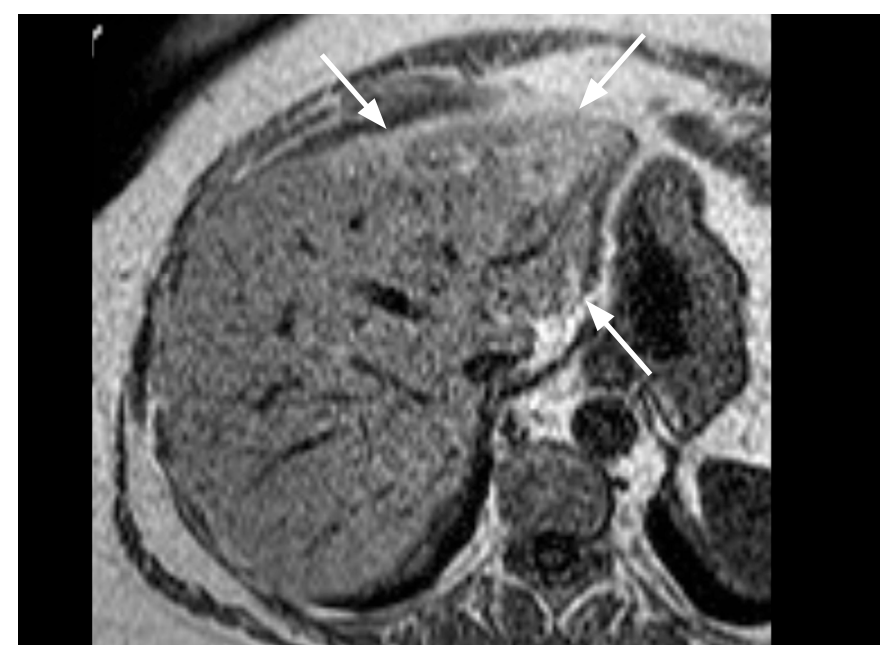

C

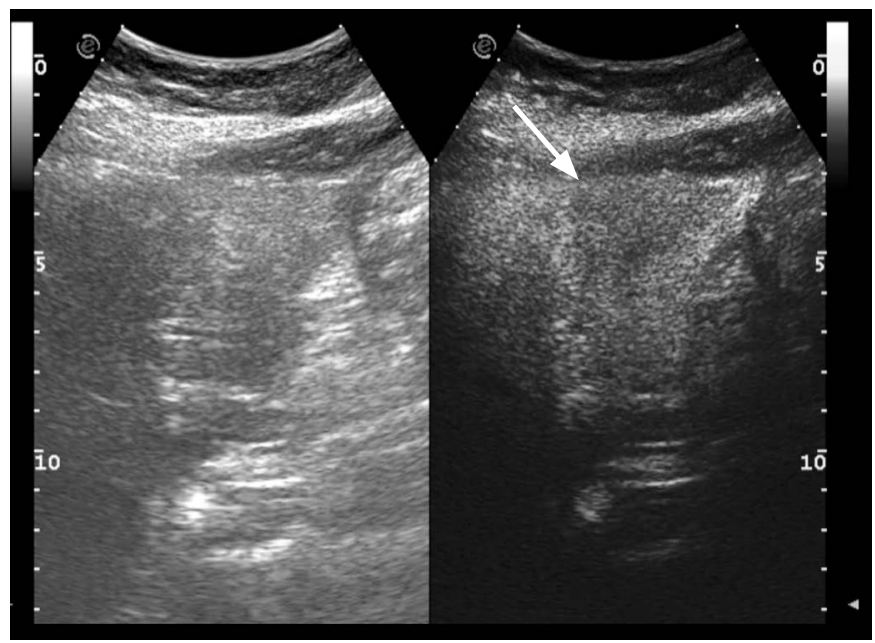

B

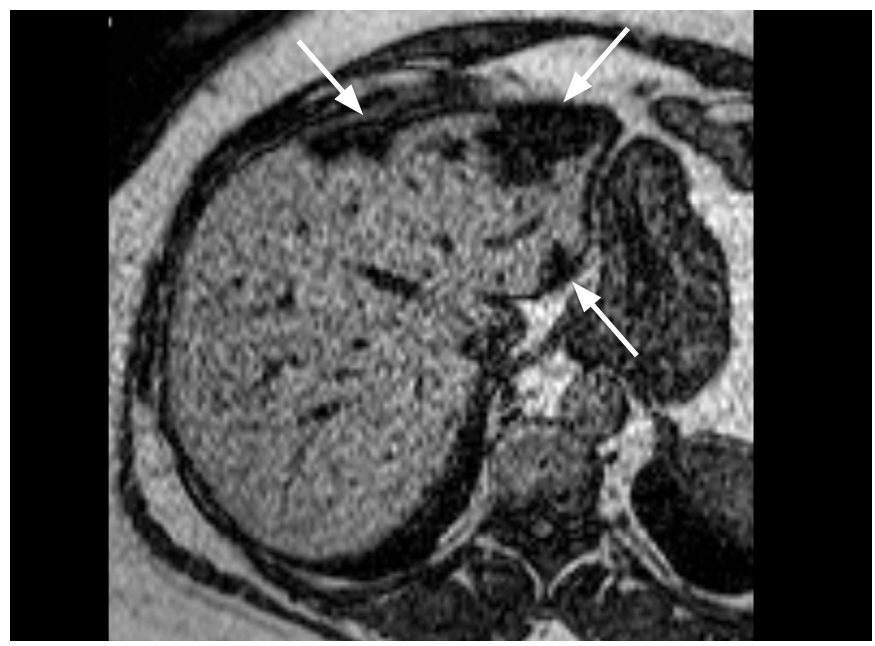

D

Fig. 4. A 63-year-old woman affected by endometrial cancer.

A. Parasagittal baseline ultrasonography shows geographic fatty change in the left hepatic lobe (arrow). B. Contrast-enhanced ultrasonography shows a hypovascular area during the extended portal-venous phase (right side, arrow) misdiagnosed as metastasis. C, D. Unenhanced magnetic resonance image confirms geographic fatty change of the liver by depicting multiple hyperintense areas (arrows) of hepatic parenchyma on T1-weighted "in-phase" image (C) with a clear-cut signal drop-out in T1-weighted "out of phase" image (arrows) (D).

remaining $32 / 37$ patients $(86.5 \%, \mathrm{P}<0.05)$ (Fig. 1).

On a patient-by-patient basis, sensitivity, specificity, positive and negative predictive values, and accuracy of CEUS versus US, considering MRI as SOR, were 100\% (95\% confidence interval [CI], 1.000 to 1.000$), 97.1 \%(95 \% \mathrm{Cl}, 0.914$ to 1.027$), 75 \%, 100 \%$, and $97.3 \%$, respectively. Inter-observer agreement was very good, with a Cohen's kappa value of $0.8426(95 \% \mathrm{Cl}, 0.5382$ to 1.00$)$.

\section{Lesion-by-Lesion Analysis}

CEUS detected all of the four metastases (size range, 5 to 10 $\mathrm{mm}$; mean, $6.7 \mathrm{~mm}$ ) confirmed by MRI. All the metastases were hypoechoic in the portal-venous phase (Fig. 2). The MRI revealed three additional lesions: one cyst $(4 \mathrm{~mm})$ in patient number 1 of Table 1 and one capillary hemangioma and one cyst, each $6 \mathrm{~mm}$ in size, both undiscovered at CEUS in a woman aged 46.8 years with ovarian adenocarcinoma (Fig. 3). Finally, in 1/37 patients (2.7\%), a 63-year-old woman with endometrial cancer, CEUS misdiagnosed geographic fatty change mainly involving liver segments $\mathrm{II}$ III, and IVa-sized 13, 40, and $46 \mathrm{~mm}$, respectively-as three metastases (Fig. 4).

In a lesion-by-lesion analysis, the difference for both CEUS and MRI versus baseline US in the detection of focal liver lesions was statistically significant $(P<0.001)$, whereas no statistically significant differences were found between CEUS versus MRI $(P=0.480)$. No adverse events were registered in our patients during or immediately 
after the injection of contrast agent.

\section{Discussion}

In the present study, we focused on the detection of liver metastases during follow-up of cancer patients without any evidence of liver metastasis, but with geographic liver steatosis at baseline US. In this particular patient population, followed-up on average for more than 4 years, we found liver metastases at CEUS in a low but not negligible percentage of patients $(8.1 \%)$, which is comparable to the lower end of the reported detection rates of liver metastases by CEUS ( $8 \%$ to $77 \%$ ) $[2,3,7,15]$. An increase of sensitivity ranging from $4 \%$ up to $107 \%$ for the detection of liver metastases compared with baseline US has also been demonstrated by using CEUS $[16,17]$.

However, there is no agreement about the usefulness of CEUS for screening and staging of metastases, and results in published studies are inconclusive, with some studies showing a similar diagnostic accuracy between CEUS and computed tomography/ MR imaging for the detection of liver metastases, while others find a significantly lower accuracy $[2,3,7,15-20]$. In our series, CEUS was able to detect all the metastases confirmed by MRI in patients with an inconclusive US study, thus leading to a dramatic change in patient management, ranging from radiofrequency thermal ablation to retreatment with chemotherapy. MRI detected no further metastases, but three benign lesions ( 2 cysts and 1 capillary hemangioma) even smaller than the metastases (mean size, 5.3 $\mathrm{mm}$ vs. $6.7 \mathrm{~mm}$ ). These findings, in spite of the observation that benign lesions are frequently encountered even in cancer patients, especially when smaller than $1.5 \mathrm{~cm}$, suggest that it is mandatory to rule out liver metastases in cancer patients, even during prolonged follow-up and when lesions are smaller than $1 \mathrm{~cm}[21,22]$. The 6-mm hemangioma missed by CEUS in our study was located in the fatty area of the liver and was isoechoic both on B-mode US and on CEUS in the portal-venous phase, thus appearing indistinguishable from the adjacent liver parenchyma. One explanation for the two small, undetected cysts $(6 \mathrm{~mm}$ and $4 \mathrm{~mm}$ ) might be a potential blooming effect in the contrast agent that strongly enhanced the adjacent hepatic tissue, but further studies addressing this issue should be undertaken.

In addition, fatty change of the liver, often occurring in cancer patients undergoing chemotherapy and presenting with different patterns of deposition and sparing, may further hamper the proper assessment of these patients by means of US $[2,12,14]$. In particular, geographic fatty liver disease is a frequently encountered variant, sometimes occurring in the right lobe of the liver and related to lipogenic alimentary factors or to an insult to the liver parenchyma, such as cholangitis or chemotherapy [5]. In a study, CEUS showed a significantly improved sensitivity compared to unenhanced US $(82.0 \%$ vs. $60.3 \%)$ for the detection of liver metastases from colorectal carcinoma after chemotherapeutic pretreatment $[23,24]$. In the present study, all the detected lesions were located in the fatty area of the liver. Although MRI performed slightly better than CEUS in the detection of liver lesions, no statistically significant difference was found, thus confirming the importance of CEUS in this particular clinical setting. In particular, the high negative predictive value of CEUS observed in our series (97\%), which is quite similar to that reported by Rafaelsen et al. [3] (98\%), suggests a role for CEUS as an effective first-line tool for ruling out the presence of metastases in cancer patients with inconclusive US study due to geographic fatty change of the liver, thus leaving the role of secondary problem solving to the more expensive procedure of MRI with liver-specific contrast agents $[3,17,25,26]$.

Interestingly, in our series, CEUS misdiagnosed geographic fatty change mainly involving liver segments II, III, and IVa as liver metastases, because of the mildly hypoechoic appearance in the portal-venous phase. Although uncommon patterns of contrastenhancement of either fatty change or focal sparing in the liver have already been reported, to our knowledge this is the first report of an area of fatty change of the liver showing a hypoechoic pattern in the portal-venous phase $[8,11,27]$. This finding is partly related to the strict criteria adopted for malignancy in this study, where any hypoenhancing area in the portal-venous phase was considered malignant. Actually, the wedge shape, the subcapsular location, lack of mass effect, and undisturbed vessel traversing through the lesion could have suggested the correct diagnosis in a real clinical setting. Further studies are needed to address this issue, especially in oncologic patients, considering that false positive cases may include necrotic tissue, fibrotic scar, peliosis, hemangiomas, adenomas, and focal nodular hyperplasia $[19,28]$. However, our study confirms the already reported high inter-observer agreement in the assessment of liver by means of CEUS [27].

The main limitation of this study is that the final diagnosis was established in the majority of cases without pathological evaluation. However, all the lesions were well characterized at multiphase contrast-enhanced MRI on the basis of typical contrastenhancement patterns considered as established diagnostic criteria. Diffusion-weighted imaging (DWI) was not performed, which is an important sequence in the modern MRI liver protocol, although in one study Gd-EOB-DTPA-enhanced MRI showed higher accuracy in the detection of small metastases than DWI [29].

A selection bias is present in our study, since we only evaluated patients suitable for US. Nevertheless, this bias does not affect the results of the present study, but reflects the limitation of US in general. For the purpose of this work, in order to ensure the best 
evaluation of both liver lobes, we decided to split the US contrast bolus into two consecutive injections with a 15-minute interval. In clinical practice, a single injection of $2.4 \mathrm{~mL}$ of contrast agent usually suffices to accomplish this task, thus sparing time and reducing costs even more [11].

In conclusion, our results show that sulphur hexafluorideenhanced US improves the diagnostic performance of US in the detection of liver metastases in cancer patients with geographic liver fatty deposition but without any evidence of metastatic disease of the liver on conventional greyscale US. In this particular clinical setting, CEUS might be of immediate practical value for daily clinical practice and of substantial importance for patient care, with its use freeing resources and helping to optimize patient management by sparing time and avoiding unnecessary and costly MR scans.

ORCID: Tommaso Vincenzo Bartolotta: http://orcid.org/0000-0002-8808-379X; Dario Picone: http://orcid.org/0000-0001-6719-7969; Massimo Midiri: http://orcid.org/00000003-1824-7549

\section{Conflict of Interest}

No potential conflict of interest relevant to this article was reported.

\section{References}

1. Figueredo A, Rumble RB, Maroun J, Earle CC, Cummings B, McLeod $R$, et al. Follow-up of patients with curatively resected colorectal cancer: a practice guideline. BMC Cancer 2003;3:26.

2. Quaia E, D'Onofrio M, Palumbo A, Rossi S, Bruni S, Cova M. Comparison of contrast-enhanced ultrasonography versus baseline ultrasound and contrast-enhanced computed tomography in metastatic disease of the liver: diagnostic performance and confidence. Eur Radiol 2006;16:1599-1609.

3. Rafaelsen SR, Jakobsen A. Contrast-enhanced ultrasound vs multidetector-computed tomography for detecting liver metastases in colorectal cancer: a prospective, blinded, patient-by-patient analysis. Colorectal Dis 2011;13:420-425.

4. Schima W, Kulinna C, Langenberger $\mathrm{H}, \mathrm{Ba}$-Ssalamah A. Liver metastases of colorectal cancer: US, CT or MR? Cancer Imaging 2005;5 Spec No A:S149-156.

5. Decarie PO, Lepanto L, Billiard JS, Olivie D, Murphy-Lavallee J, Kauffmann $C$, et al. Fatty liver deposition and sparing: a pictorial review. Insights Imaging 2011;2:533-538.

6. Larsen LP. Role of contrast enhanced ultrasonography in the assessment of hepatic metastases: a review. World J Hepatol 2010;2:8-15.

7. Bartolotta TV, Midiri M, Galia M, Rollandi GA, Cademartiri F, Lagalla $R$, et al. Characterization of benign hepatic tumors arising in fatty liver with SonoVue and pulse inversion US. Abdom Imaging
2007;32:84-91.

8. Janica J, Ustymowicz A, Lukasiewicz A, Panasiuk A, NiemcunowiczJanica A, Turecka-Kulesza E, et al. Comparison of contrast-enhanced ultrasonography with grey-scale ultrasonography and contrastenhanced computed tomography in diagnosing focal fatty liver infiltrations and focal fatty sparing. Adv Med Sci 2013;58:408-418.

9. Bartolotta TV, Taibbi A, Galia M, Runza G, Matranga D, Midiri M, et al. Characterization of hypoechoic focal hepatic lesions in patients with fatty liver: diagnostic performance and confidence of contrastenhanced ultrasound. Eur Radiol 2007;17:650-661.

10. World Medical Association. Declaration of Helsinki. Ethical principles for medical research involving human subjects [Internet]. FerneyVoltaire: World Medical Association, 2016 [cited 2016 Dec 1]. Available from: http://www.wma.net/en/30publications/10policies/ b3/.

11. Claudon M, Dietrich CF, Choi BI, Cosgrove DO, Kudo M, Nolsoe CP, et al. Guidelines and good clinical practice recommendations for contrast enhanced ultrasound (CEUS) in the liver: update 2012: a WFUMB-EFSUMB initiative in cooperation with representatives of AFSUMB, AIUM, ASUM, FLAUS and ICUS. Ultraschall Med 2013;34:11-29.

12. Hamer OW, Aguirre DA, Casola G, Lavine JE, Woenckhaus M, Sirlin CB. Fatty liver: imaging patterns and pitfalls. Radiographics 2006;26:1637-1653.

13. Lee HY, Lee JM, Kim SH, Shin KS, Lee JY, Han JK, et al. Detection and characterization of focal hepatic lesions: comparative study of MDCT and gadobenate dimeglumine-enhanced MR imaging. Clin Imaging 2008;32:287-295.

14. Bartolotta TV, Taibbi A, Midiri M, Lagalla R. Focal liver lesions: contrast-enhanced ultrasound. Abdom Imaging 2009;34:193-209.

15. Mainenti PP, Mancini M, Mainolfi C, Camera L, Maurea S, Manchia $\mathrm{A}$, et al. Detection of colo-rectal liver metastases: prospective comparison of contrast enhanced US, multidetector $\mathrm{CT}, \mathrm{PET} / \mathrm{CT}$, and 1.5 Tesla MR with extracellular and reticulo-endothelial cell specific contrast agents. Abdom Imaging 2010;35:511-521.

16. Bertolotto M, Vicenzo Bartolotta T, Belgrano M, Trincia E, Cernic $S$, Zappetti $R$, et al. Detection of liver metastases with contrast enhanced ultrasonography. Curr Med Imaging Rev 2007;3:37-44.

17. Nicolau C, Ripolles T. Contrast-enhanced ultrasound in abdominal imaging. Abdom Imaging 2012;37:1-19.

18. Larsen LP, Rosenkilde M, Christensen H, Bang N, Bolvig L, Christiansen $\mathrm{T}$, et al. Can contrast-enhanced ultrasonography replace multidetector-computed tomography in the detection of liver metastases from colorectal cancer? Eur J Radiol 2009;69:308-313.

19. Muhi A, Ichikawa T, Motosugi U, Sou H, Nakajima H, Sano K, et al. Diagnosis of colorectal hepatic metastases: comparison of contrastenhanced CT, contrast-enhanced US, superparamagnetic iron oxideenhanced MRI, and gadoxetic acid-enhanced MRI. J Magn Reson Imaging 2011;34:326-335. 
20. Cabassa P, Bipat S, Longaretti L, Morone M, Maroldi R. Liver metastases: sulphur hexafluoride-enhanced ultrasonography for lesion detection: a systematic review. Ultrasound Med Biol 2010;36:1561-1567.

21. van Erkel AR, Pijl ME, van den Berg-Huysmans AA, Wasser MN, van de Velde CJ, Bloem JL. Hepatic metastases in patients with colorectal cancer: relationship between size of metastases, standard of reference, and detection rates. Radiology 2002;224:404-409.

22. Khalil HI, Patterson SA, Panicek DM. Hepatic lesions deemed too small to characterize at CT: prevalence and importance in women with breast cancer. Radiology 2005;235:872-878.

23. Bartolotta TV, Vernuccio F, Taibbi A, Lagalla R. Contrast-enhanced ultrasound in focal liver lesions: where do we stand? Semin Ultrasound CT MR 2016;37:573-586.

24. Konopke R, Bunk A, Kersting S. Contrast-enhanced ultrasonography in patients with colorectal liver metastases after chemotherapy. Ultraschall Med 2008;29 Suppl 4:S203-S209.

25. Kulemann V, Schima W, Tamandl D, Kaczirek K, Gruenberger T, Wrba
$\mathrm{F}$, et al. Preoperative detection of colorectal liver metastases in fatty liver: MDCT or MRI? Eur J Radiol 2011;79:e1-e6.

26. Bartolotta TV, Taibbi A, Midiri M, La Grutta L, De Maria M, Lagalla R. Characterisation of focal liver lesions undetermined at grey-scale US: contrast-enhanced US versus 64-row MDCT and MRI with liverspecific contrast agent. Radiol Med 2010;115:714-731.

27. Bartolotta TV, Taibbi A, Midiri M, Matranga D, Solbiati L, Lagalla $R$. Indeterminate focal liver lesions incidentally discovered at grayscale US: role of contrast-enhanced sonography. Invest Radiol 2011;46:106-115.

28. Kim TK, Jang HJ, Burns PN, Murphy-Lavallee J, Wilson SR. Focal nodular hyperplasia and hepatic adenoma: differentiation with low-mechanical-index contrast-enhanced sonography. AJR Am J Roentgenol 2008;190:58-66.

29. Shimada K, Isoda H, Hirokawa Y, Arizono S, Shibata T, Togashi K. Comparison of gadolinium-EOB-DTPA-enhanced and diffusionweighted liver MRI for detection of small hepatic metastases. Eur Radiol 2010;20:2690-2698. 